\title{
An autopsy case of ectopic ACTH-secreting lung carcinoid with Cushing's syndrome.
}

Yui Watanabe, Koichiro Hara, Kei-ichiro Matoba, Hiroyuki Yamazaki, Katsuyoshi Tojo, Kazunori Utsunomiya

Division of Diabetes, Metabolism and Endocrinology, Department of Internal Medicine, the Jikei University School of Medicine

\section{Case Report}

【Chief Complaint】 reduction in appetite and weakness of lower limbs

【Onset and course】

A 81-year-old woman came to our hospital with complaints of reduction in appetite and weakness of lower limbs. She had developed Cushing's features. Initial laboratory evaluation revealed severe hypokalemia, so she were admitted to our hospital for further examination.

【Past History】 Uterus myoma

【Familv Historv】 Unremarkable

【Physical examination】

Height $137 \mathrm{~cm}$, Weight $47 \mathrm{~kg}$, BMI $25.0 \mathrm{~kg} / \mathrm{m}^{2}$ Blood Pressure 112/80mmHg,

Pulse Rate 102/min, regular

She had moon face, buffalo hump and skin pigmentation.

Conjunctive was not anemic and not icteric

Pulmonary, cardiac and neurological examinations were unremarkable.

She had pitting edema in her legs.

\section{Laboratory Findings}

\begin{tabular}{|c|c|c|c|c|c|}
\hline$\angle C B C>$ & & <Bioc & & & \\
\hline & $9500 / \mu \mathrm{L}$ & AST & $29 \mathrm{U} / \mathrm{L}$ & & $206 \mathrm{mg} / \mathrm{dL}$ \\
\hline & $94.3 \%$ & & $46 \mathrm{U} / \mathrm{L}$ & & \\
\hline & 年.3\% & 甚H & $446 \mathrm{U} / \mathrm{L}$ & $\begin{array}{l}\text { Anti-GAD Antibody } \\
\text { CFA }\end{array}$ & $<0.3 \mathrm{U} / \mathrm{mL}$ \\
\hline & $\begin{array}{l}1.4 \% \\
0.0 \%\end{array}$ & A APP & $218 \mathrm{U} / \mathrm{L}$ & $\begin{array}{l}\text { CYFRA } \\
\text { ChPA }\end{array}$ & $\begin{array}{l}12.1 \mathrm{ng} / \mathrm{mL} \\
8.0 \mathrm{ng} / \mathrm{mL}\end{array}$ \\
\hline $\mathrm{BCC}$ & & VGT & $82 \mathrm{U} / \mathrm{h}$ & ProGRP & $281.0 \mathrm{pg} / \mathrm{mL}$ \\
\hline & $8.7 \mathrm{~g} / \mathrm{dL}$ & & $4.2 \mathrm{~g} / \mathrm{dL}$ & & $17.1 \mathrm{ng} / \mathrm{mL}$ \\
\hline ncv & $\begin{array}{l}26.4 \% \\
92.3 \mathrm{fL}\end{array}$ & All & $\begin{array}{ll}2.1 \mathrm{~g} / \mathrm{dL} \\
32 \mathrm{mg} / \mathrm{dL}\end{array}$ & & \\
\hline мсHC & $30.4 \%$ & $\mathrm{cr}$ & $0.66 \mathrm{mg} / \mathrm{dl}$ & & \\
\hline & $\times 10^{4} / \mu \mathrm{L}$ & UA & $2.1 \mathrm{mg} / \mathrm{dL}$ & Glu & $\begin{array}{l}1+11 \\
(+3)\end{array}$ \\
\hline & $1.3 \%$ & $\mathrm{Na}$ & $144 \mathrm{mmol} / \mathrm{L}$ & Ket & \\
\hline APTT & $26.7 \mathrm{sec}$ & $\mathrm{cl}$ & $105 \mathrm{mmol} / \mathrm{L}$ & $\begin{array}{l}\text { Bil } \\
\text { Uro }\end{array}$ & \\
\hline & & CRP & 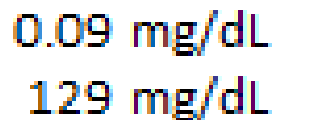 & OB & ${ }^{(+2)}$ \\
\hline & & & $38 \mathrm{mg} / \mathrm{dL}$ & & $30-49 / \mathrm{HPF}$ \\
\hline
\end{tabular}

<Endocrinological examinations>

$\begin{array}{lll}\mathrm{GH} & 0.15 \mathrm{ng} / \mathrm{mL} & \text { ACTH } \\ & 32 \mathrm{ng} / \mathrm{mL} & \mathrm{F}\end{array}$

FSH
PRL
TSH
FH

$0.10 \mathrm{~m} / \mathrm{u} / \mathrm{mL} \quad$ DHEA-S

DHEA
PRA
PAC

$11.44 \mathrm{pg} / \mathrm{mL}$

$0.06 \mu \mathrm{llU} / \mathrm{m}$

FT4 $\quad 0.9 \mathrm{ng} / \mathrm{dL}$

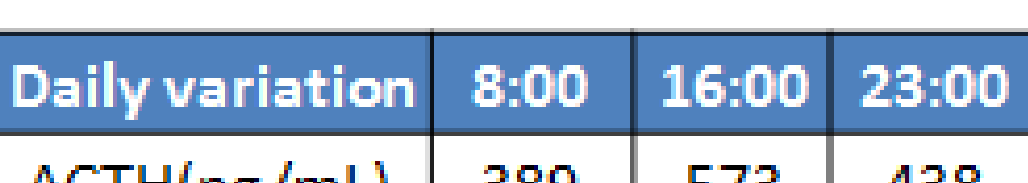

\begin{tabular}{|c|c|c|}
\hline CRH test & ACTH(pg/mL) & F( $(\mu / \mathrm{g} / \mathrm{dL})$ \\
\hline Pre & 628 & 140 \\
\hline $15 \mathrm{~min}$ & 946 & 160 \\
\hline $30 \mathrm{~min}$ & 889 & 151 \\
\hline $60 \mathrm{~min}$ & 736 & 141 \\
\hline $90 \mathrm{~min}$ & 569 & 166 \\
\hline $120 \mathrm{~min}$ & 559 & 149 \\
\hline
\end{tabular}

There was loss of Daily variation.

ACTH response was normal.

\section{Discussions}

【Examinations for differentiate ectopic ACTH syndrome from Cushing's disease】

\begin{tabular}{|c|c|c|}
\hline Examinations & sensitivity & specificity \\
\hline HDDST & $80-94 \%$ & $80-94 \%$ \\
\hline CRH test & $90-100 \%$ & $85 \%$ \\
\hline IPSS & $90 \%$ & $67 \%$ \\
\hline Y3-MSH & unknown & unknown \\
\hline
\end{tabular}

Imaging Test

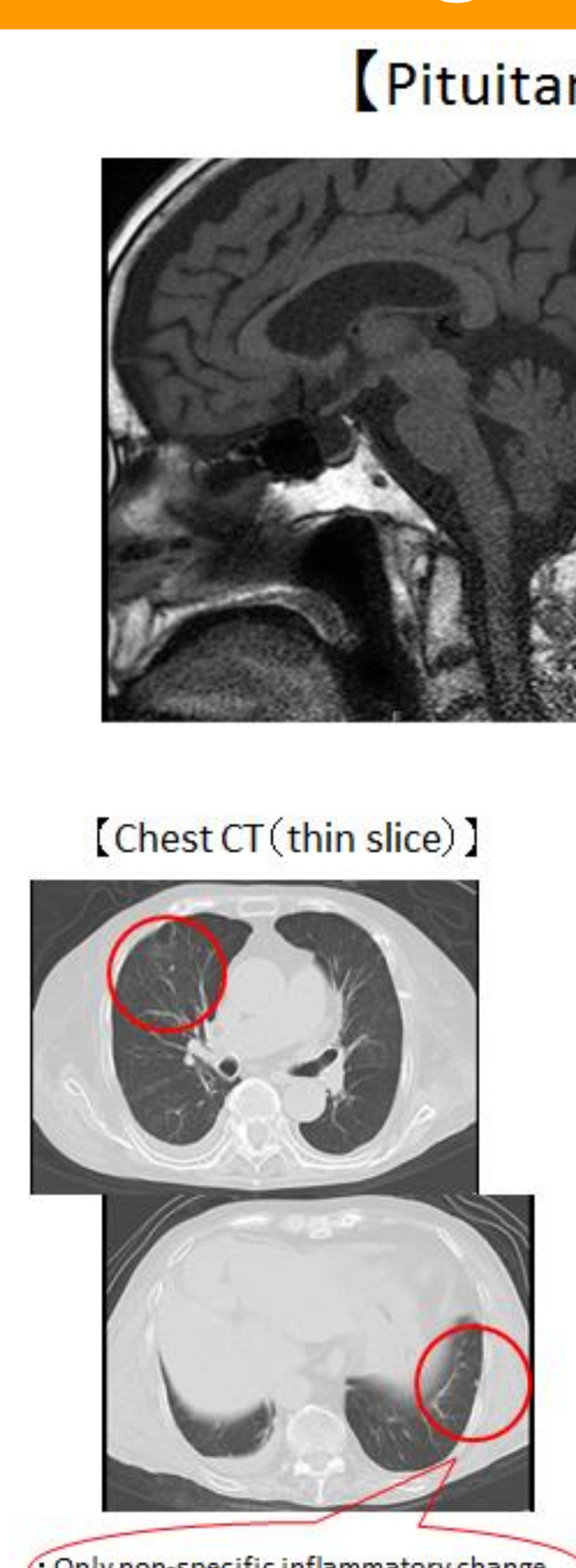

【FDG PET/CT】

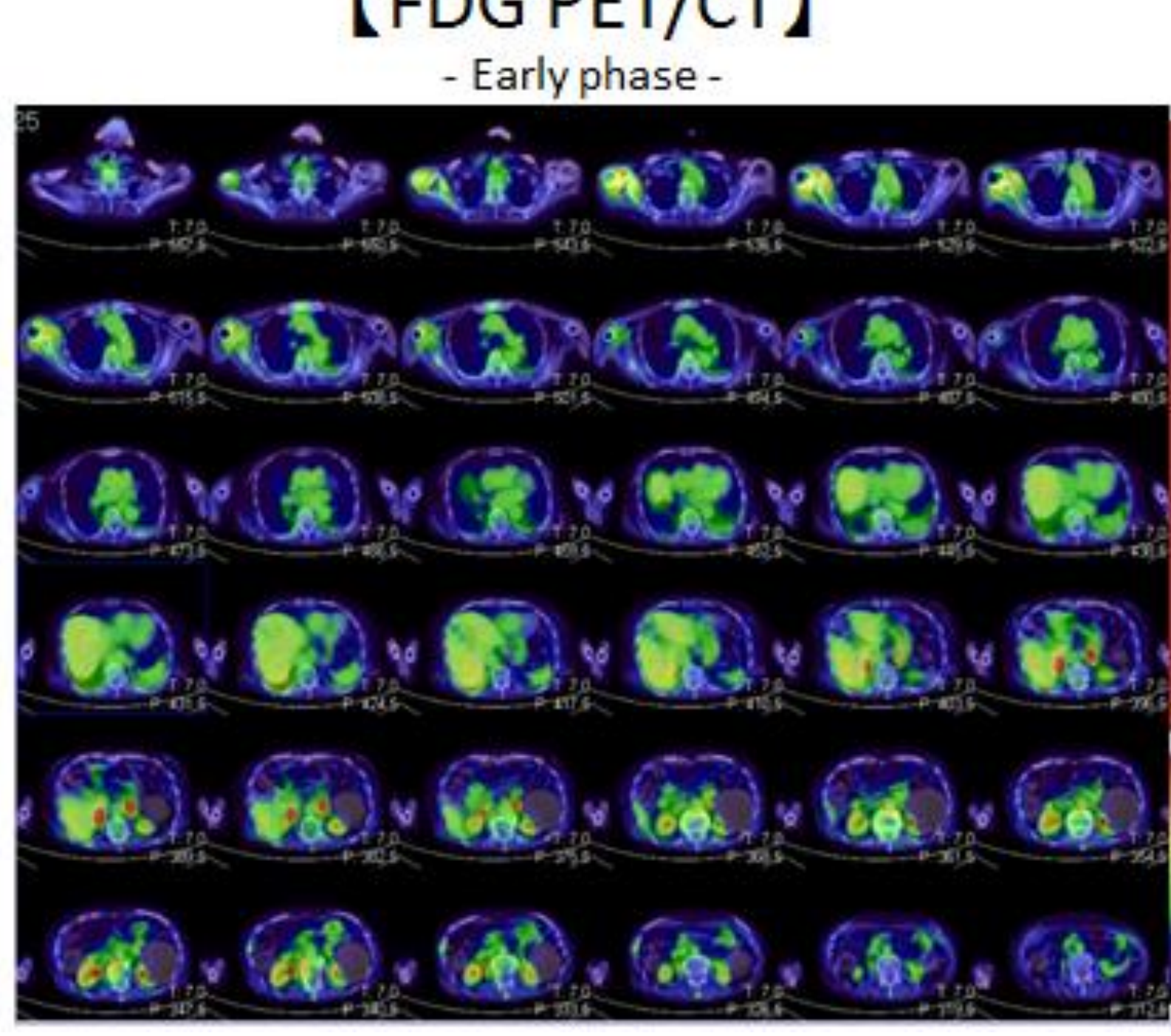

Delay phase

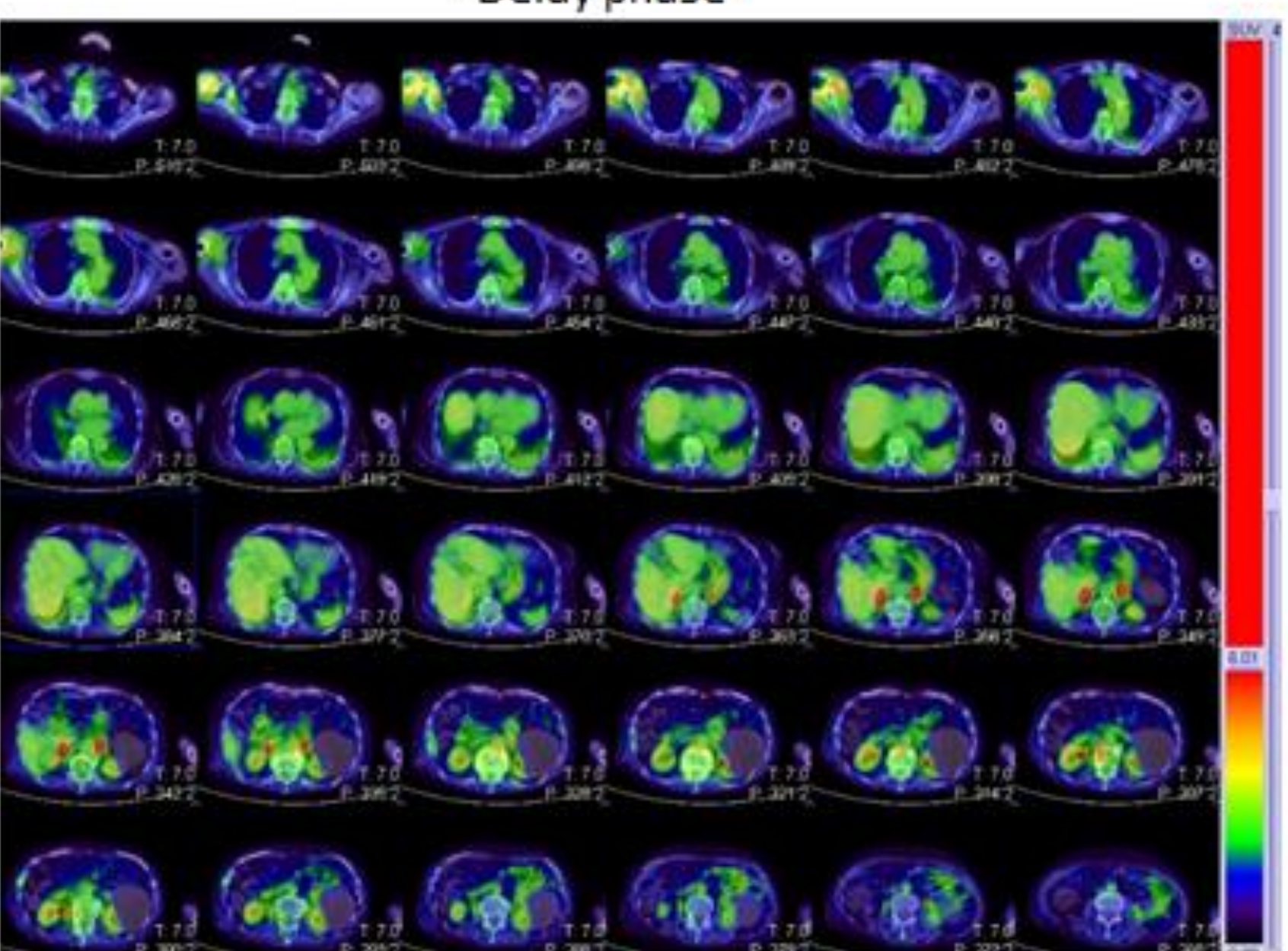

- No obviviustumor

【Localization diagnosis for ectopic ACTH syndrome】

\begin{tabular}{|c|c|}
\hline Examinations & $\begin{array}{c}\text { positive predictive } \\
\text { value }\end{array}$ \\
\hline Chest CT/MRI & $68 \%(25 / 37$ case $)$ \\
\hline $\begin{array}{c}\text { somatostatin receptor } \\
\text { scintigraphy }\end{array}$ & $60 \%(8 / 12$ case $)$ \\
\hline FDG-PET & $66 \%(4 / 6$ case $)$ \\
\hline $\begin{array}{c}\text { (for lung cartinoid) } \\
\text { Lung artery sampling }\end{array}$ & unknown \\
\hline
\end{tabular}

Crinical Course

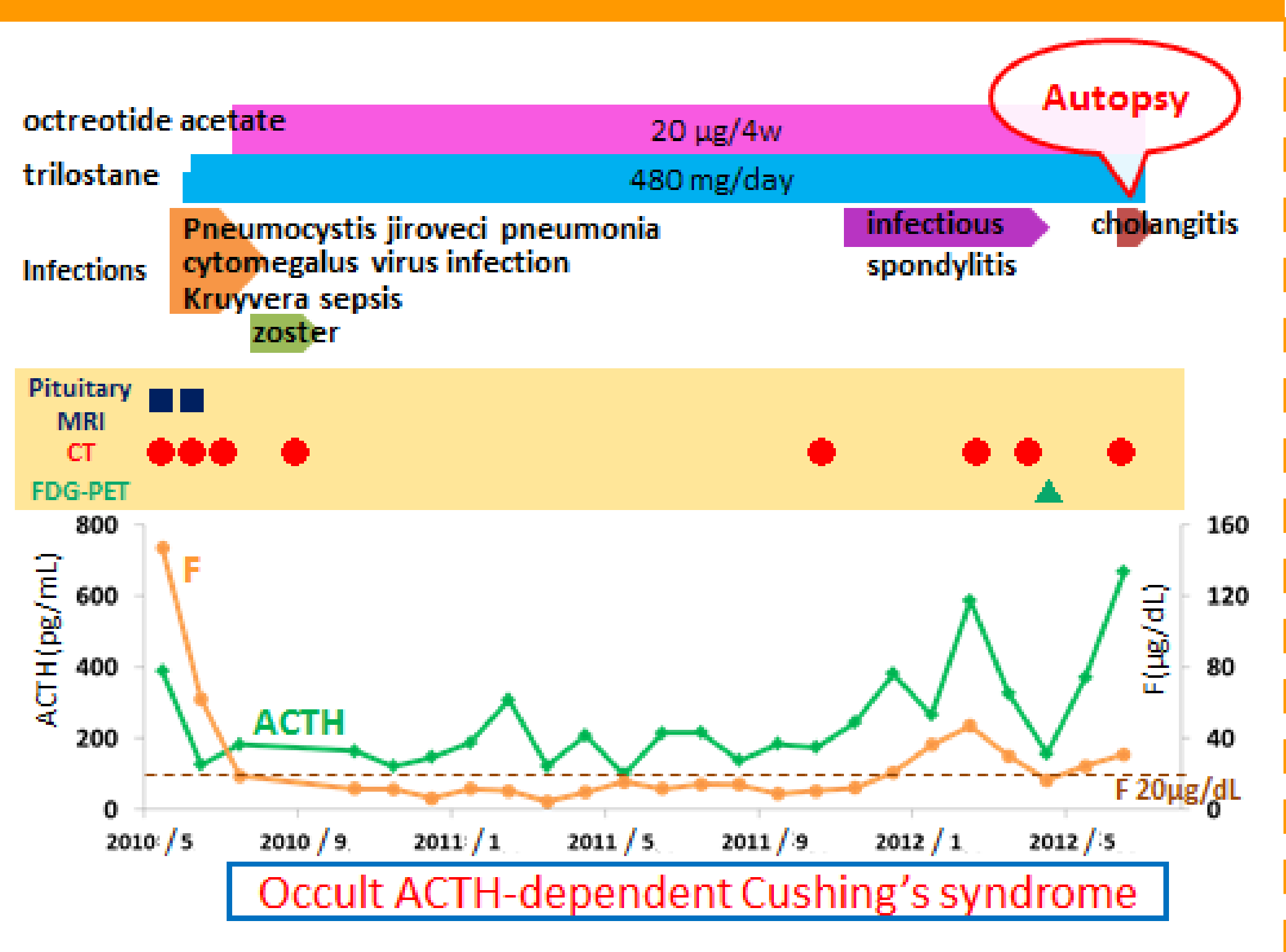

\section{Pathological Findings}

Pituitary> HE $\times 4$

915g

<Lung tumor> macroscopic picture
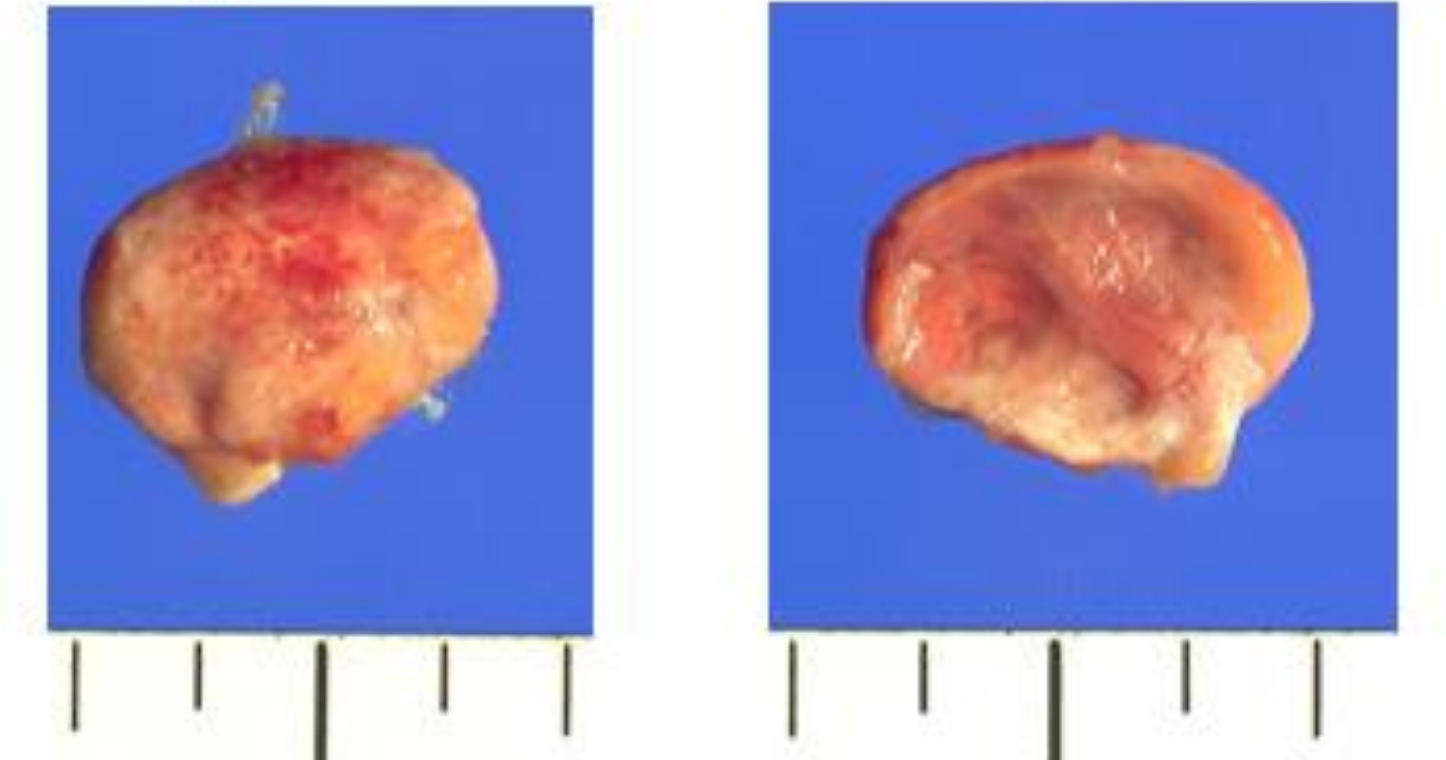

Left lower lobe, $17 \mathrm{~mm}$ diameter

$\times 40$

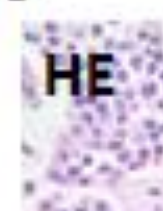

HE.

chromogranin $A$.

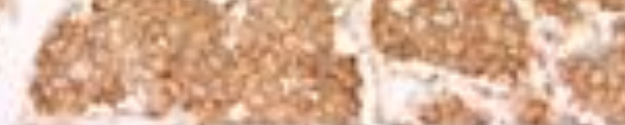

synaptophysin

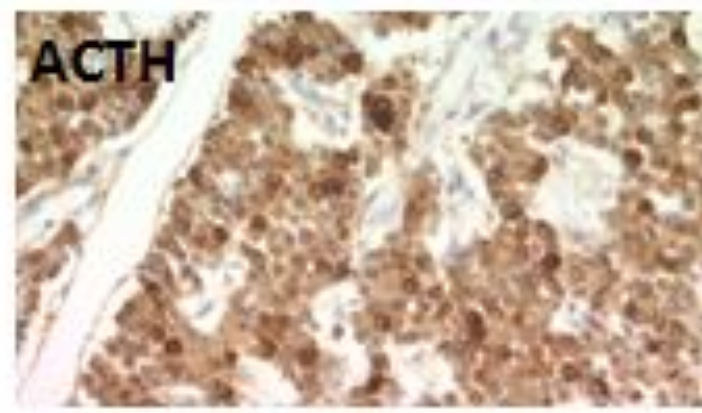

ectopic ACTH-secreting lung carcinoid

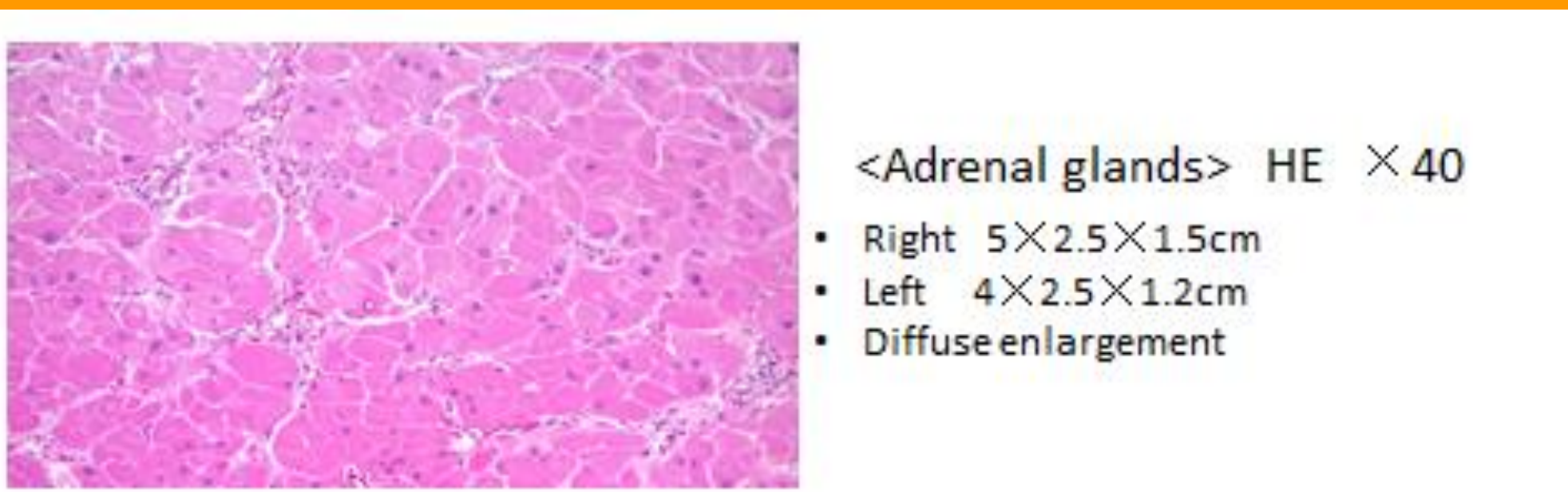

\section{Conclusion}

- We experienced a case of lung carcinoid that we could not detect a localization regardless of repeated radiological imaging, but autopsy confirmed its diagnosis.

- For localizing primary lesion of ACTHsecreting tumor, repeatedly and fully imaging tests is very important. 